\title{
Why people living with HIVIAIDS exclude individuals from their chosen families
}

\author{
This article was published in the following Dove Press journal: \\ Nursing: Research and Reviews \\ II March 2013 \\ Number of times this article has been viewed
}

\author{
Joan S Grant' \\ David E Vance' \\ Worawan White ${ }^{2}$ \\ Norman L Keltner' \\ James L Raper ${ }^{3}$ \\ 'School of Nursing, University \\ of Alabama at Birmingham, \\ Birmingham, Alabama, ${ }^{2}$ Department \\ of Nursing, Pensacola State College, \\ Pensacola, Florida, ${ }^{3}$ School \\ of Medicine, Department of Medicine, \\ Division of Infectious Diseases, \\ University of Alabama at Birmingham, \\ Birmingham, Alabama, USA
}

\begin{abstract}
Health professionals can gain a better understanding of key elements of social support by examining reasons why people living with human immunodeficiency virus/acquired immunodeficiency syndrome (PLWH) exclude individuals from their chosen families (ie, families of choice). Our study identified reasons why PLWH excluded specific individuals from their chosen families. This mixed-method design was drawn from a larger study of 150 PLWH, in which 94 self-reported why they excluded individuals from their chosen families. Physical and emotional distance $(\mathrm{n}=64 ; 68.1 \%)$; nonsupport, nonacceptance, and harm $(\mathrm{n}=25 ; 26.6 \%)$; conditional caring and trust $(\mathrm{n}=22 ; 23.4 \%)$; and no blood/familial relationship $(\mathrm{n}=13 ; 13.8 \%$ ) were the reasons PLWH excluded individuals from their chosen families. Demographic and personal characteristics were unrelated to these themes, supporting the conclusion that reasons for excluding family members are universal and not dependent on particular participant characteristics. For chosen family relationships to develop and exist, these findings emphasize the value of physical and emotional contact between individuals.
\end{abstract}

Keywords: families, stigma, social networks, human immunodeficiency virus, acquired immunodeficiency syndrome

\section{Introduction}

Infection with the human immunodeficiency virus (HIV) is at epidemic levels and people living with HIV/acquired immunodeficiency syndrome (AIDS) (PLWH) may experience depression, stress, anger at their diagnosis, fear of disease progression, and worries about infecting others. Unfortunately, these factors are also associated with negative physiological and psychological outcomes, such as a decreased quality of life, nonadherence to care, faster disease progression, and increased mortality. ${ }^{1-3}$ Unlike for people with many other chronic health conditions, PLWH are also faced with issues related to disclosure and potential stigma and discrimination. ${ }^{4-6}$

For some, their family is a major source of support. In fact, the family unit is the most important social unit in most countries of the world. Family life is important in the sense that it gives individuals basic strength. Therefore, for PLWH, their family often influences how well they manage significant challenges associated with this disease. While family and friends can be significant sources of social support, ${ }^{7}$ PLWH may also fear isolation and associated stigma and discrimination from them. Some feel betrayed, neglected, avoided, or shunned by those they expected to stand by them - their "family.", 8

Individuals who are marginalized due to stigma create alternative family structures for support and companionship in lieu of families related by blood or marriage. 
Therefore, many gay, lesbian, bisexual, and transgender individuals have chosen individuals other than those who are related by blood or marriage to provide them with social support. Therefore, in this paper, "family" is defined more broadly to include biologically related members and other social relationships, such as partners, friends, and relatives. $^{9-12}$

A review of the literature failed to reveal any published empirical literature examining why PLWH exclude individuals from their chosen families. However, health professionals can gain a better understanding of key elements of social support by examining the reasons why PLWH exclude individuals from their chosen families (ie, families of choice). Our study identified reasons why PLWH excluded specific individuals from their chosen families.

While there was no available literature related to this specific topic, other studies regarding family structure, disclosure, and stigma added insight to this investigation. The chosen families of PLWH offer social support, love, and affection and are held together by inspiration, commitment, caring, and trust. Families also provide stability, structure, inspiration, mentorship, and encouragement to PLWH. Chosen families are sources of functional social support that offer emotional support by asking PLWH about their general well-being, giving general encouragement, and by talking with them about daily issues and challenges (but not necessarily about their HIV status). Instrumental support is provided through household and financial assistance (eg, food, shelter, monies)..$^{13,14}$

Grant et al offered further insight into why chosen family members are important to PLWH. Using a mixedmethod design of a convenience sample of $150 \mathrm{PLWH}$ aged 19-68 years old, they reported reasons why PLWH included specific individuals in their chosen families. ${ }^{11}$ Self-reported reasons for including specific individuals in their chosen families were love and acceptance $(n=135$; $90.0 \%$ ), support $(\mathrm{n}=100 ; 66.7 \%)$, blood and family ties $(\mathrm{n}=37 ; 24.7 \%)$, and commonality $(\mathrm{n}=28 ; 18.7 \%)$. These findings emphasize the importance of relationships between PLWH and those who provide love and acceptance, support, blood/familial ties, and have common interests.

Further, Serovich et al examined reasons why 48 HIV-positive men who have sex with men (MSM) felt their families of origin (through blood, adoption, and marriage) were important. ${ }^{15}$ Participants were recruited from a large Midwestern city in the USA. Family as a unique relationship was a significant reason why MSM felt their families of origin were important, emphasizing the value of biological ties.
Contact and proximity were also an important factor. While participants had more consistent exchanges with their friends, they also had significant close relationships with biological families. Family closeness or the loving nature of the biological family was yet another reason why their families of origin were important to MSM and significantly influenced whether they disclosed their serostatus to their relatives. However, a quarter of the MSM also had a family of creation that was at least as important as, if not more important than, their biological family to them.

Because social support is important to both physical and mental health, Peterson examined social support challenges of women living with HIV. ${ }^{8}$ A qualitative approach was used to interview 45 women living with HIV/AIDS. Participants were recruited from three cities in the US Midwest. These participants encountered numerous challenges in seeking and receiving social support. The women feared abandonment by family members and that their families would not keep their status private. Many were worried that telling their family would result in others knowing about their diagnosis. The women also recounted instances in which they were treated differently by their family (eg, separate eating utensils, a specific place to sit, and limited or no physical contact with children). The women also balanced their need for social support with overburdening their families. In disclosing to children, these participants often failed to share their HIV status for fear of worrying them or causing them sadness.

In examining disclosure to specific family members, Serovich et al examined 135 HIV-positive MSM. ${ }^{16}$ These participants were drawn from a larger longitudinal study of HIV disclosure regarding mental and physical health, social support, disease progression, and sexual risk-taking behaviors. Of the 597 family members of participants, 50\% had been informed of their HIV status. Participants disclosed their HIV status in greater proportion to their mother $(77 \%)$ than to others: $47 \%$ to fathers, $50 \%$ to sisters, and $41 \%$ to brothers. After accounting for characteristics of participants and family members, there was no difference in the rate at which family members were told. In examining patterns of support in PLWH, Bor et al suggested that it was not the type of relationship that facilitated talking about HIV status, but, rather, other qualities such as openness and emotional closeness. ${ }^{17}$ Further, knowing that a person was not prejudiced might simplify to whom MSM with HIV confide about their HIV status.

In another study, Paiva et al examined disclosure of HIV-positive serostatus by 250 heterosexual and bisexual men to sexual partners. ${ }^{18}$ Fear of rejection led to isolation 
and distress, thus hindering disclosure to current and new partners. HIV/AIDS diagnosis was more commonly disclosed to steady partners, partners who were HIV-positive themselves, and female partners. These investigators suggested stigma management is an important element underlying personal motivation that must be considered while enhancing comprehensive care for HIV-positive men.

Gaskins et $\mathrm{al}^{19}$ also examined reasons for disclosure and nondisclosure of HIV status among 40 rural AfricanAmerican men in the Southern USA. From audiotaped interviews, the authors determined that the most common reasons this sample disclosed to other people were to relieve their stress, satisfy their need to tell, to help others, and to receive support. The most common reasons for not disclosing their status were a fear of negative reactions or stigma from others, worrying the disclosure recipient would tell others, a belief that there was no need to tell, not being ready to tell, and not wanting to burden others with their disclosure.

Latkin et $\mathrm{al}^{20}$ also examined correlates of disclosure of MSM and seropositive HIV status to individuals in their social networks. Data for the study were drawn from a cohort of African-American MSM recruited for a pilot HIV risk-reduction intervention in Baltimore, Maryland. In these 187 African-American MSM, disclosure of MSM behavior to social network members was more common among participants who were younger, had a higher educational level, and were HIV infected. Participants were more likely to disclose their MSM behavior to people in their social networks who were HIV infected, gave them emotional support, socialized with them, and were not a female sex partner. Younger participants were more likely to disclose their positive HIV status to those who were older, HIV infected, provided emotional support, loaned them money, and were not a male sex partner.

In a review of the HIV disclosure literature, Obermeyer et $\mathrm{al}^{21}$ also found that few people kept their status completely secret. Stigmatization increased the fear of disclosure and disclosure tended to be a gradual process, with more PLWH revealing their HIV status to those in their social networks over time. Disclosure also appeared to be higher in the USA and Europe. Sharing positive HIV status to partners varied greatly but appeared to be lower with casual partners than with steady partners. Further, disclosure to relatives appeared to be higher than to friends.

Their survey of people living with $\mathrm{HIV}^{21}$ found that stigma in family settings - in particular, avoidance, exaggerated kindness, and being told to conceal one's status - was a significant predictor of psychological distress. This was believed to be due to the absence of unconditional love and support that families are expected to provide. Furthermore, people living with HIV are often worried about losing family and friends if they disclose their status.

Finally, Serovich et $\mathrm{a}^{22}$ examined the prevalence of regret related to the disclosure of serostatus and sexual orientation to family members among HIV-positive MSM. They also explored participant, family member, and relationship characteristics that influenced the likelihood of experiencing regret over disclosure. Almost half of participants indicated no regret in disclosing either their HIV-positive serostatus or sexual orientation. Among those who did experience some regret, the prevalence of regret over disclosing to one or more family members was similar for HIV-positive serostatus (39.4\%) and for sexual orientation (37.3\%).

Parker and Aggleton ${ }^{23}$ emphasized how stigma and discrimination, as social processes, strengthen and reproduce existing inequalities of gender, race, and social class. For example, in addition to stigma around HIV and homosexuality faced by PLWH, regional factors such as higher rates of poverty; racism that helps drive and fuel the problem; cultural conservatism that serves as a barrier to sensitization/education; stigma toward drug addiction, prostitution and promiscuity; and sexism play an important role in higher incident rates of HIV in the US South. In addition, religious or moral beliefs lead some people to believe that being infected with HIV is the result of moral fault (such as promiscuity or "deviant sex") that deserves to be punished.

Stigma arises and develops within the context of culture and power, and is used by individuals, communities, and others to produce and reproduce inequality. Parker and Aggleton $^{23}$ suggest new agendas for research and action, focusing on conceptual studies that examine social, cultural, political, and economic causes and consequences of stigmatization and discrimination. Further, these authors recommend developing our understanding of the social processes at work in HIV and the ways that these social processes influence HIV vulnerability. ${ }^{23}$

In summary, because of the isolation, rejection, and stigmatization that are usually associated with HIV/AIDS, some PLWH become distant from their traditional families. However, PLWH manage to create chosen families that include those they consider family. Proximity, support, love and acceptance, and biological ties appear to be important in defining the chosen families of PLWH. HIV disclosure appeared to a gradual process and more PLWH disclosed to those who provided emotional support and socialized with them. 


\section{Participants and methods}

This investigation was drawn from a larger study of 150 PLWH in the southeastern USA examining relationships of individuals who PLWH included in their chosen families as well as why PLWH either include or exclude specific individuals from their chosen families. Relationships of individuals who PLWH included in their chosen families and reasons why PLWH include these specific individuals in their chosen families are reported elsewhere. ${ }^{7,11}$ In the present study, using a mixed-method design, content analysis was used to identify why PLWH exclude individuals from their chosen families and quantitative analysis was used to report their most common reasons. Of the larger sample, 94 out of the 150 PLWH gave reasons for excluding specific individuals from their chosen families. Demographic and personal characteristics were also examined to determine if these reasons were associated with identified themes for excluding an individual as a family member.

\section{Participants}

Study approval was obtained from The University of Alabama at Birmingham (Birmingham, AL, USA) institutional review board and study facilities; this included an approved written consent form for participants to sign. In this study, a convenience sample was recruited from two HIV outpatient clinics in the southeastern USA. Inclusion criteria included being a male or nonpregnant female with a self-reported diagnosis of HIV; 19 years of age or older; and able to speak, read, and write English. Further, participants also were cognitively unimpaired, as indicated by a score $>10$ on the HIV Dementia Scale. ${ }^{24}$ Of this subsample $(n=94)$, participants ranged in age from 19 to 61 years old and were primarily male (81.9\%) and African-American (70.2\%). Participants' self-described sexual orientation was almost equally heterosexual (52.1\%) and homosexual/bisexual/ other $(47.9 \%)$.

\section{Procedures}

Posters and flyers describing the study were posted in each clinic to recruit study participants. Physicians and nurse practitioners in the clinics also shared basic information about the study with their patients. Interested potential participants subsequently contacted the investigators to learn more about the study. A trained research nurse interviewed potential participants to explain the study, verify eligibility, and secure informed consent prior to completing study questionnaires.

Eligible participants completed an investigator-developed questionnaire privately in a private room in one of the outpatient clinics where they sought care. Following questionnaire completion, participants were given US $\$ 10$ for their time and participation. Questionnaire completion was assessed prior to participants leaving the clinic.

\section{Measures}

\section{Cognitive status}

The HIV Dementia Scale ${ }^{24}$ was used to screen PLWH' cognitive status to determine their eligibility to be enrolled in the study. Scores on this instrument range from 0 to 16, with a cut-off of 10 or less suggesting HIV-associated cognitive impairment or dementia. This scale has good internal consistency (Cronbach's alpha $=0.75)$.

\section{Sociodemographic and family data questionnaire}

An investigator-developed questionnaire was used to assess sociodemographic characteristics, individuals PLWH included or did not include in their chosen families, and reasons why they included or excluded them from their families. The sociodemographic section of the questionnaire included questions concerning age, gender, race, education, sexual orientation, employment and income status, and HIV-related information (eg, duration of HIV infection, current viral load and HIV medications, emotional/mental problems, and recent opportunistic infections). The questionnaire contained a list of persons with whom participants could potentially have a relationship. This list included relatives by blood or marriage (eg, fathers, mothers, children, brothers and sisters, spouses, relatives, in-laws, and grandparents) as well as other individuals not related by blood or marriage (eg, friends, dates, partners, sexual partners [lovers]). Using this list of existing relationships, participants were asked to indicate individuals they included or did not include as a member of their family of choice and provide a rationale for this inclusion and exclusion. Participants checked "Does not apply" when a respective person was non-applicable (eg, death of a parent, grandparent). Data analyses for this article focus on individuals who PLWH did not include in their chosen families.

The appropriateness and clarity of the instrument items were evaluated by three experts who collectively had expertise in family dynamics, psychometrics, and HIV/AIDS. Using two rounds of a modified Delphi technique, all content experts agreed regarding the appropriateness and clarity of instrument items, supporting the instrument's content validity.

\section{Data analysis}

Data were analyzed using SPSS (v 14.0; IBM, Armonk, NY). Measures of central tendency and variability, frequencies, and 
percentages were used to quantify reasons participants excluded individuals from their chosen families. Only participants with existing relationships were included in the analyses. Demographic and personal characteristics (ie, gender, race) were examined to determine if they were statistically related to excluding specific individuals from their chosen families.

Prior to analyzing the quantitative data, a conventional content analysis ${ }^{25}$ was conducted. Similar codes were grouped together and aggregated under a single inclusive definition for the broader conceptual meaning of why PLWH excluded individuals from their chosen families. Two research team members with backgrounds in HIV, psychometrics, and family dynamics generated descriptive codes for emerging themes in the data and refined those codes until the team reached consensus that the terms were adequate for defining, explaining, and categorizing reasons why participants excluded specific individuals from their family of choice.

Once the coding scheme was determined, all transcripts were independently reviewed a second time and coded in their entirety according to the newly developed coding scheme. To ensure coding reliability, the research team met to discuss coding interpretations. Coding discrepancies were discussed until consensus about the appropriate code was obtained. Another team member randomly selected $10 \%$ of the transcripts and recoded them independently again in terms of the existing descriptive codes. In comparing the coding of participants' responses between this team member and the other investigators, the Cohen's kappa coefficient was 0.94 . Validity was addressed by checking transcripts against themes and interpretations.

\section{Results}

Of the sample, 94 of the 150 PLWH identified specific persons they excluded from their chosen families and gave reasons for excluding them. Descriptive statistics regarding the 94 participants are presented in Table 1 and trends shown in this table reflect the larger overall sample of 150 participants. Participants' ages ranged from 19 to 61 years with a mean age of 40.4 years ( standard deviation $=8.0$ ). They were primarily single $(\mathrm{n}=85 ; 90.4 \%)$, male $(\mathrm{n}=77 ; 81.9 \%)$, and African-American $(\mathrm{n}=66 ; 71.3 \%)$; had completed high school education or more ( $\mathrm{n}=46 ; 48.9 \%)$; were on disability $(\mathrm{n}=48 ; 51.1 \%)$ and unemployed $(\mathrm{n}=73 ; 77.7 \%)$; had an annual income of $<$ US $\$ 10,000(n=71 ; 75.5 \%)$; and reported no current emotional/mental problems $(n=62$; $66.0 \%)$. The number of participants identifying themselves as heterosexual $(\mathrm{n}=49 ; 52.1 \%)$ was comparable to those
Table I Characteristics of participants who provided reasons for including/excluding individuals from their chosen families $(\mathrm{N}=94)$

\begin{tabular}{|c|c|c|c|}
\hline Characteristic & Mean (SD) & $\mathbf{N}$ & $\%^{\mathrm{a}}$ \\
\hline Age (years) & $40.4(8.0)$ & & \\
\hline \multicolumn{4}{|l|}{ Gender } \\
\hline Male & & 17 & \\
\hline Female & & 77 & \\
\hline \multicolumn{4}{|l|}{ Sexual orientation } \\
\hline Heterosexual & & 49 & 52.1 \\
\hline Homosexual & & 34 & 36.2 \\
\hline Bisexual and/or other & & II & 11.7 \\
\hline \multicolumn{4}{|l|}{ Race } \\
\hline Caucasian (non-Hispanic) & & 23 & 24.5 \\
\hline African-American & & 66 & 70.2 \\
\hline Other (eg, Asian, Hispanic) & & 5 & 5 \\
\hline \multicolumn{4}{|l|}{ Current marital status } \\
\hline Single & & 63 & 67.0 \\
\hline Married & & 4 & 4.3 \\
\hline Live with partner & & 10 & 10.6 \\
\hline Divorced & & 5 & 5.3 \\
\hline Other & & 12 & 12.8 \\
\hline \multicolumn{4}{|l|}{ Current employment status } \\
\hline Employed part-time & & 11 & 11.7 \\
\hline Employed full-time & & 10 & 10.6 \\
\hline Unemployed & & 24 & 25.5 \\
\hline On disability & & 48 & 51.1 \\
\hline Other & & I & 1.1 \\
\hline Highest education achieved (years) & $12.4(2.4)$ & & \\
\hline Less than high school & & 23 & 24.5 \\
\hline Completed high school & & 25 & 26.6 \\
\hline Some college & & 28 & 29.8 \\
\hline College graduate & & 7 & 7.4 \\
\hline Graduate degree & & I & 1.1 \\
\hline Others (eg, training certificates) & & 10 & 10.6 \\
\hline \multicolumn{4}{|l|}{ Annual income (USD) } \\
\hline$\leq \$ 10,000$ & & 71 & 75.5 \\
\hline$\$ 10,001-\$ 29,999$ & & 19 & 20.2 \\
\hline$\geq \$ 30,000$ & & 4 & 4.3 \\
\hline
\end{tabular}

Note: asum of percentages may be $>100$ due to rounding.

Abbreviation: SD, standard deviation.

identifying themselves as homosexual ( $\mathrm{n}=34 ; 36.2 \%)$. Further, eleven (11.7\%) individuals specified an "other" (eg, bisexual) sexual preference.

Participants had an average 9.1 years (standard deviation $=5.5$ ) of known HIV-positive status. Most selfreported they were prescribed at least one HIV medication $(\mathrm{n}=73,77.7 \%)$ and had had no opportunistic infections during the previous 3 months ( $\mathrm{n}=68 ; 72.3 \%$ ). The average CD4+ lymphocyte count and viral load of the sample was 364.5 cells $/ \mathrm{mm}^{3}(\mathrm{SD}=258.1)$ and 56,529 copies $/ \mathrm{mL}$ ( $\mathrm{SD}=141,442.0)$, respectively. However, approximately one-third ( $\mathrm{n}=29 ; 30.9 \%)$ of participants had an undetectable viral load $(<50$ copies $/ \mathrm{mL})$ and approximately one-third $(\mathrm{n}=28 ; 29.8 \%)$ had AIDS (ie, a CD4+ lymphocyte count 
of $\leq 200$ cells $\left./ \mathrm{mm}^{3}\right)$. Most $(\mathrm{n}=89 ; 94.7 \%)$ had revealed their HIV status to someone in their lives.

\section{Themes}

Physical and emotional distance $(n=64 ; 68.1 \%)$; nonsupport, nonacceptance, and harm $(\mathrm{n}=25 ; 26.6 \%)$; conditional caring and trust $(\mathrm{n}=22 ; 23.4 \%)$; and no blood/familial relationship $(\mathrm{n}=13 ; 13.8 \%$ ) were the four reasons PLWH excluded individuals from their chosen families. While $65(69.1 \%)$ participants voiced one of these reason themes, $28(29.8 \%)$ participants voiced two themes and one participant (69.1\%) voiced three themes.

\section{Physical and emotional distance}

The most frequent reason for PLWH excluding individuals from their chosen families was physical and emotional distance. PLWH excluded these individuals because of a limited or lack of tangible and affective closeness. Further, this reason was often given because the contact and sharing of specific information, such as their HIV-positive serostatus, was considered irrelevant to those individuals they excluded from their chosen families. Specific responses included:

- "We have no contact at all."

- "We are not and have never been close."

- "Disowned me growing up, didn't care what happened to me."

- "Don't see them. They don't know if I am dead or alive."

- "None of their business."

- "Don't want them to know my status [...]."

- "Just don’t need to know."

- “It isn't easy to talk about, not close so I don't tell them everything."

\section{Nonsupport, nonacceptance, and harm}

PLWH excluded individuals from their chosen families for providing them no support or not accepting their homosexuality and HIV-positive serostatus. A lack of understanding between PLWH and particular individuals, and rejection, was inherent in this exclusion. Further, there was dissonance or disagreement between PLWH and others regarding acceptance of their homosexuality and HIV-positive serostatus. Those excluded from their family of choice included both biological and nonbiological relations. Some participants cited physical and sexual harm to themselves in previous years as a reason for exclusion of specific individuals. Therefore, rejection of the participant as a person, their homosexuality, and HIV status was the major and underlying reason for excluding specific individuals as part of their chosen families. Specific responses included:

- "He is not good to me (raped me when I was young)."

- “One sister loved me but didn't like the color I was dating and my HIV status."

- " "... they react differently to people with HIV."

- "They are not understanding at all."

- "Smothering, they both know, I have explained but they don't understand what HIV is."

- "Not gay friendly, they react to me differently b/c [because] of my being gay."

- "I don't include my brother because he's a snob."

- "Rejects and acts differently to me."

\section{Conditional caring and trust}

Whereas the previous theme was based on actual nonsupport, nonacceptance, and harm, participants also cited many reasons for excluding an individual from their chosen families because of tentative or conditional emotional closeness and trust. PLWH feared negative consequences, such as rejection or loss of a relationship or employment, if these individuals knew their HIV status. They also feared that others would be told of their HIV status if they made it known to specific individuals they excluded from their chosen families. Specific responses included:

- “Not open minded, doesn't know about my HIV, but wouldn't accept it."

- "They don't know about my HIV, afraid of them telling other people."

- "I don't trust them."

- "They have no knowledge; they might break up the friendship."

- "They don't know my status; they would reject me because they have no knowledge."

- "Afraid of being stereotyped and ..."

- "They don't know, don't want to expect or experience a negative reaction."

- “She doesn't know and I haven't told her. I'm afraid she would not be able to take it and get disappointed."

\section{No blood/familial relationship}

PLWH also excluded certain individuals from their chosen families because there was no blood or familial tie. Therefore, a lack of kinship was an important reason to exclude someone from their family. For example, some friends were excluded because they were not related by blood. Further, although participants had positive relationships with friends, participants sometimes excluded specific friends 
because of a lack of kinship. Individuals with whom PLWH had relationships that were at an early stage or were for sexual gratification were also not viewed by PLWH as members of their chosen families. Specific responses included:

- "They are not blood related."

- "They are friends, not blood related."

- "I care for them, but not like my family."

- "We only see each other once per week, pretty much just sex."

- "They are just friends, not a family."

- "Not blood related despite a good and close relationship."

- "They're friends, associates, not blood related ..."

- "It is just a casual (superficial relationship)."

\section{Themes expressed by groups}

In follow-up analyses using the entire sample of 150 participants, demographic and personal characteristics were examined to determine if they were associated with excluding individuals from being chosen family members. In Table 2, the demographic and personal characteristics of the sample are compared, using chi-squares, according to whether they identified with any of the four themes. Only one demographic was related by theme: those who were employed were more likely to voice physical and emotional distance as a reason for excluding someone from being a member of their chosen family $\left(\chi^{2}[\mathrm{~N}=150]=7.7, P=0.005\right)$. However, using a Bonferroni correction (alpha of 0.05 divided by 40 tests $=$ alpha correction of 0.00125 ), this relationship was no longer significant. Therefore, based upon the lack of significant findings between demographic and personal characteristics and expressed themes, the findings suggest that these themes may be universal to human behavior and not dependent on particular participant characteristics.

\section{Discussion}

The purpose of this study was to identify why PLWH exclude individuals from their chosen families. The most common reasons PLWH excluded individuals from their chosen families were physical and emotional distance; followed by nonsupport, nonacceptance, and harm; conditional caring and trust; and no blood/familial relationship. Demographic and personal characteristics (ie, gender, race) were not related to the exclusion of individuals from their chosen families. These themes emphasize that distance, both emotional and physical, and a lack of support, acceptance, caring, and trust are reasons why PLWH exclude other individuals from their chosen families. Further, a portion of this sample excluded individuals from their chosen family based upon whether they were related by blood or marriage, reinforcing the value of these ties.

These findings are similar to those cited by Gaskins et al for not disclosing HIV status, including fear of negative reactions, worrying that disclosure recipients would tell others, belief that there was no need to tell, and not wanting to burden others. ${ }^{19}$ In contrast, our study findings also emphasize essential components for disclosing HIV-positive status to others. Similar to reasons suggested by Bor et al, ${ }^{17}$ we found that PLWH need an open, emotionally close, and unprejudiced relationship with others to trust them enough to disclose their HIV status.

While these findings indicate reasons for excluding individuals from chosen families, they also have implications for health professionals in terms of appropriate behaviors when interacting with and providing care to PLWH. These findings emphasize the importance of health professionals demonstrating supportive behaviors that indicate they value and accept PLWH. Further, interactions should be frequent enough to build therapeutic relationships that are built upon caring and trust.

Moreover, basic counseling skills are essential to address the stigma experienced by PLWH. Stigma management programs are important tools in this respect, as they lessen negative attitudes and beliefs about HIV. ${ }^{18}$ Given Peterson's ${ }^{8}$ findings, who cited examples of how PLWH were treated differently by their family (ie, those related to them by blood or marriage) when they disclosed their status to them, the development of family counseling and support programs to improve the physical and psychological health of individuals living with HIV and their family is essential.

Balaji et $\mathrm{al}^{26}$ concurred in their study of the role of familial, religious, and community influences on the experiences of young black MSM. Their results suggest that homosexuality remains highly stigmatized in the men's families, religious communities, and the African-American community. To manage stigma, many participants used "role flexing," in which they changed their behavior to adapt to a situation. ${ }^{26}$ Thus, interventions should focus on social, political, and economic causes of stigma and stigmatization and concentrate on aggregates and communities rather than just on individuals. Further, priority should be given to intervention programs that focus on communities developing new models for advocacy and social change. These programs should be part of a multidimensional effort, with structural and environmental interventions directed at transforming the context in which both individuals and communities operate as they respond to HIV. ${ }^{26,27}$ 
Table 2 Themes expressed by grouping variables $(N=150)$

\begin{tabular}{|c|c|c|c|c|}
\hline Grouping variable & $\begin{array}{l}\text { Physical and emotional } \\
\text { distance }(n=64)\end{array}$ & $\begin{array}{l}\text { Nonsupport, nonacceptance, } \\
\text { and harm }(n=25)\end{array}$ & $\begin{array}{l}\text { Conditional caring } \\
\text { and trust }(n=22)\end{array}$ & $\begin{array}{l}\text { No blood/familial } \\
\text { relationship }(n=13)\end{array}$ \\
\hline \multicolumn{5}{|l|}{ Age $^{a}$ (years) } \\
\hline$<40(\mathrm{n}=56)$ & 24 (42.9\%) & 12 (2I.4\%) & $10(17.96 \%)$ & $6(10.7 \%)$ \\
\hline$\geq 40(n=94)$ & $40(42.6 \%)$ & $13(13.8 \%)$ & $12(12.8 \%)$ & $7(7.4 \%)$ \\
\hline \multicolumn{5}{|l|}{ Gender } \\
\hline Male $(n=118)$ & 47 (39.8\%) & $24(20.3 \%)$ & $19(16.1 \%)$ & II (9.3\%) \\
\hline Female $(n=31)$ & 17 (54.8\%) & I (3.2\%) & $3(9.7 \%)$ & $2(6.5 \%)$ \\
\hline Transgendered $(n=I)$ & $0(0 \%)$ & $0(0 \%)$ & $0(0 \%)$ & $0(0 \%)$ \\
\hline \multicolumn{5}{|l|}{ Sexual orientation } \\
\hline Heterosexual $(n=7 I)$ & 34 (47.9\%) & $13(18.3 \%)$ & $14(19.7 \%)$ & 8 (11.3\%) \\
\hline Homosexual $(n=65)$ & $24(36.9 \%)$ & $8(12.3 \%)$ & $7(10.8 \%)$ & $3(4.6 \%)$ \\
\hline Other $(n=14)$ & $6(42.9 \%)$ & $4(28.6 \%)$ & I (7.I\%) & $2(14.3 \%)$ \\
\hline \multicolumn{5}{|l|}{ Race } \\
\hline African-American $(\mathrm{n}=105)$ & $43(41.0 \%)$ & $15(14.3 \%)$ & $18(17.1 \%)$ & $10(76.9 \%)$ \\
\hline Caucasian $(n=39)$ & $20(51.3 \%)$ & $8(20.5 \%)$ & $3(7.7 \%)$ & $2(5.1 \%)$ \\
\hline Other $(n=6)$ & I (I6.7\%) & $3(33.3 \%)$ & I $(0.7 \%)$ & I (I6.7\%) \\
\hline \multicolumn{5}{|l|}{ Marital status } \\
\hline Single $(n=132)$ & $55(41.7 \%)$ & $23(17.4 \%)$ & $19(14.4 \%)$ & I3 (9.8\%) \\
\hline Coupled $(n=18)$ & $9(50.0 \%)$ & $2(11.1 \%)$ & $3(16.7 \%)$ & $0(0 \%)$ \\
\hline \multicolumn{5}{|l|}{ Highest education } \\
\hline High school or less $(n=8 \mathrm{I})$ & $29(35.8 \%)$ & $13(16.9 \%)$ & $13(16.0 \%)$ & $8(9.9 \%)$ \\
\hline Higher than high school $(n=69)$ & $35(50.7 \%)$ & $12(17.4 \%)$ & $9(13.0 \%)$ & $5(7.2 \%)$ \\
\hline \multicolumn{5}{|l|}{ Current employment status } \\
\hline Employed $(n=27)$ & $18(66.7 \%)^{\mathrm{a}}$ & $3(11.1 \%)$ & $5(18.5 \%)$ & $2(7.4 \%)$ \\
\hline Unemployed $(n=123)$ & $46(37.6 \%)$ & $22(17.9 \%)$ & $17(13.8 \%)$ & II (8.9\%) \\
\hline \multicolumn{5}{|l|}{ Annual income (USD) } \\
\hline$<\$ 10,000(n=116)$ & $48(41.4 \%)$ & $18(15.5 \%)$ & $18(15.5 \%)$ & $13(11.2 \%)$ \\
\hline$\geq \$ 10,000(n=34)$ & $16(47.1 \%)$ & $7(20.6 \%)$ & $4(11.8 \%)$ & $0(0 \%)$ \\
\hline \multicolumn{5}{|c|}{ Current emotional/mental problems } \\
\hline Yes $(n=5 I)$ & $19(37.3 \%)$ & $8(15.7 \%)$ & $7(13,7 \%)$ & $6(11.8 \%)$ \\
\hline No $(n=99)$ & $45(45.5 \%)$ & $17(17.2 \%)$ & $15(15.2 \%)$ & $7(7.1 \%)$ \\
\hline \multicolumn{5}{|c|}{ Disclose HIV status to someone } \\
\hline Yes $(n=145)$ & $63(43.4 \%)$ & $23(15.9 \%)$ & $21(14.5 \%)$ & 12 (8.3\%) \\
\hline No $(n=5)$ & I (20.0\%) & $2(40.0 \%)$ & I (20.0\%) & I (20.0\%) \\
\hline
\end{tabular}

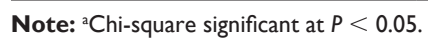

Abbreviation: HIV, human immunodeficiency virus.

Cahill et al also emphasized the necessity for other community-based HIV prevention interventions that combat prejudice against MSM and transgender women. ${ }^{27}$ Community-based prevention intervention programs that assert the healthy formation of gay and transgender identities are necessary. These investigators also recognized the value of gay-affirming school-based interventions and resiliency-focused social-marketing campaigns that have already had a positive impact on health outcomes and should be implemented on a broader scale to address anti-gay stigma. ${ }^{27}$ One might hold out hope that, given the broadening acceptance/tolerance of marriage equality, someday the burden of homosexual stigma may also be removed along with a social cascade of factors that relate to the negativity of being infected with HIV.
Peterson also emphasized the value of physical and emotional contact between individuals for relationships among chosen families to develop and exist. ${ }^{8}$ Many individuals excluded biological family members because of distant relationships and a lack of tangible and affective closeness between themselves and others. In interacting with their chosen families, therapeutic and supportive communication between PLWH and others is important. If personal face-to-face contact is not possible, then contact by letter, email, and/or telephone may be feasible.

Our study has limitations, including the use of a cross-sectional design. Because participation was initiated by volunteers in response to study flyers and information provided by health care providers, the limitations of a self-selected sample must be recognized in this study. 
Considering these study findings have limited generalizability, future studies of other populations such as women would be valuable in examining why PLWH exclude individuals from their chosen families. Further, this study should be replicated in parts of the country other than the southeastern USA to examine the similarity of themes. Although this study did examine whether demographic and personal characteristics were related to certain themes, a more in-depth examination of such potential factors is important in future research.

\section{Conclusion}

Reasons for excluding individuals from their family of choice include a lack of physical and emotional contact; nonsupport and nonacceptance because of their HIV status as well as harm to themselves; tentative caring and trust between PLWH and others; and a lack of biological/familial ties. These study findings emphasize the potential and actual stigma and rejection PLWH face.

Health professionals have an essential role in reducing the stigma associated with HIV and improving the quality of life of PLWH by increasing awareness about this chronic disease, challenging prejudice, providing nonjudgmental care, teaching adaptive coping skills, and serving as role models. ${ }^{28}$

\section{Disclosure}

The authors report no conflicts of interest in this work. All authors made substantial contributions to this study in terms of (1) the conception and design, acquisition of data, or analysis and interpretation of data; (2) drafting the article or revising it critically for important intellectual content; and (3) giving final approval of the version to be published.

\section{References}

1. Freudenreich O, Goforth HW, Cozza KL, et al. Psychiatric treatment of persons with HIV/AIDS: an HIV-psychiatry consensus survey of current practices. Psychosomatics. 2010;51(6):480-488.

2. Horberg MA, Silverberg MJ, Hurley LB, et al. Effects of depression and selective serotonin reuptake inhibitor use on adherence to highly active antiretroviral therapy and on clinical outcomes in HIV-infected patients. J Acquir Immune Defic Syndr. 2008;47(3):384-390.

3. Leserman J. Role of depression, stress, and trauma in HIV disease progression. Psychosom Med. 2008;70(5):539-545.

4. Greene K, Banjeree SC. Disease-related stigma: comparing predictors of AIDS and cancer stigma. J Homosex. 2006;50(4):185-209.

5. Rao D, Kekwaletswe TC, Hosek S, Martinez J, Rodriguez F. Stigma and social barriers to medication adherence with urban youth living with HIV. AIDS Care. 2007;19(1):28-33.

6. Stutterheim SE, Bos AE, Pryor JB, Brands R, Liebregts M, Schaalma HP. Psychological and social correlates of HIV status disclosure: the significance of stigma visibility. AIDS Educ Prev. 2011;23(4):382-392.

7. Prachakul W, Grant JS, Pryor E, Keltner NL, Raper JL. Family relationships in people living with HIV in a city in the USA. AIDS Care. 2009;21(3):384-388.
8. Peterson JL. The challenges of seeking and receiving support for women living with HIV. Health Comm. 2010;25(5):470-479.

9. Butler SS. Gay, lesbian, bisexual and transgender (GLBT) elders: the challenges and resilience of this marginalized group. J Hum Behav Soc Environ. 2004;9(4):25-44.

10. de Vries B, Blando JA. The study of gay and lesbian aging: Lessons for social gerontology. In: Herdt G, de Vries B, editors. Gay and Lesbian Aging: Research and Future Directions. New York, NY: Springer; 2004:3-28.

11. Grant JS, Vance DE, Keltner NL, White W, Raper JL. Reasons why persons living with HIV include individuals in their chosen families. J Assoc Nurses AIDS Care. 2013;24(1):50-60.

12. Wright $F$. The role of the family as a support system for people with AIDS. Nurse Pract Forum. 1991;2(2):134-136.

13. George S, Garth B, Wohl AR, Galvan FH, Garland W, Myers HF. Sources and types of social support that influence engagement in HIV care among Latinos and African Americans. J Healthcare Poor Care Underserved. 2009;20(4):1012-1035.

14. Poindexter C, Shippy RA. Networks of older New Yorkers with HIV: fragility, resilience, and transformation. AIDS Patient Care STDS. 2008;22(9):723-733.

15. Serovich JM, Grafsky EL, Craft SM. Does family matter to HIV-positive men who have sex with men? J Marital Fam Ther. 2011;37(3):290-298.

16. Serovich JM, Esbensen AJ, Mason TL. HIV disclosure by men who have sex with men to immediate family over time. AIDS Patient Care STDs. 2005;19(8):506-517.

17. Bor R, du Plessis P, Russell M. The impact of disclosure of HIV on the index patient's self-defined family. J Fam Ther. 2004;26(2):167-192.

18. Paiva V, Segurado AC, Filipe EM. Self-disclosure of HIV diagnosis to sexual partners by heterosexual and bisexual men: A challenge for HIV/ AIDS care and prevention. Cad Saude Publica. 2011;27:1699-1710.

19. Gaskins S, Payne Foster P, Sowell R, Lewis T, Gardner A, Parton J. Reasons for HIV disclosure and non-disclosure: an exploratory study of rural African American men. Issues Ment Health Nurs. 2011;32(6): 367-373.

20. Latkin C, Yang C, Tobin K, Roebuck G, Spikes P, Patterson J. Social network predictors of disclosure of MSM behavior and HIV-positive serostatus among African American MSM in Baltimore, Maryland. AIDS Behav. 2012;16(3):535-542.

21. Obermeyer CM, Baijal P, Pegurri E. Facilitating HIV disclosure across diverse settings: a review. Am J Public Health. 2011;101(6): 1011-1023.

22. Serovich JM, Grafsky EL, Reed S. Comparing regret of disclosing HIV versus sexual orientation information by MSM. AIDS Care 2010;22(9):1052-1059.

23. Parker R, Aggleton P. HIV and AIDS-related stigma and discrimination: a conceptual framework and implications for action. Soc Sci Med. 2003;57(1):13-24.

24. Power C, Selnes OA, Grim JA, McArthur JC. HIV Dementia Scale: a rapid screening test. J Acquir Immune Defic Syndr Hum Retrovirol. 1995;8(3):273-278.

25. Hsieh HF, Shannon SE. Three approaches to qualitative content analysis. Qual Health Res. 2005;15(9):1277-1288.

26. Balaji AB, Oster AM, Viall AH, Heffelfinger JD, Mena LA, Toledo CA. Role flexing: how community, religion, and family shape the experiences of young black men who have sex with men. AIDS Patient Care STDS. 2012; 26(12):730-737.

27. Cahill S, Valadéz R, Ibarrola S. Community-based HIV prevention interventions that combat anti-gay stigma for men who have sex with men and for transgender women. J Public Health Policy. 2013:34(1): 69-81.

28. Lewis R. Stamping out stigma in HIV. Nurs Times. 2011;107(11): 16-17. 
Nursing: Research and Reviews

Dovepress

\section{Publish your work in this journal}

Nursing: Research and Reviews is an international, peer-reviewed, open access journal publishing original research, reports, reviews and commentaries on all aspects of nursing and patient care. These include patient education and counselling, ethics, management and organizational issues, diagnostics and prescribing, economics and

resource management, health outcomes, and improving patient safety in all settings. The manuscript management system is completely online and includes a very quick and fair peer-review system. Visit http://www.dovepress.com/testimonials.php to read real quotes from published authors.

Submit your manuscript here: http://www.dovepress.com/nursing-research-and-reviews-journal 